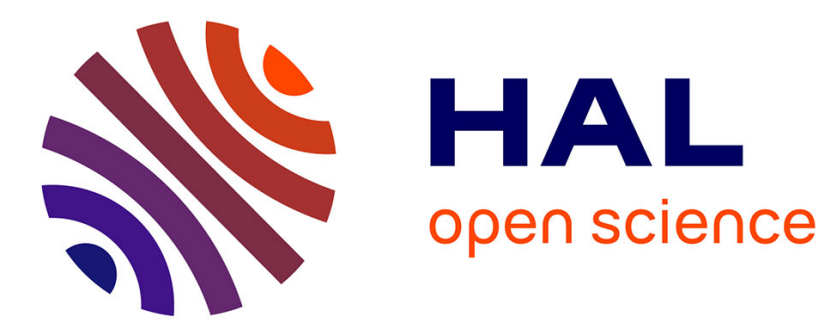

\title{
Étude acoustique de la production de voyelles de l'anglais par des apprenants francophones
} Jennifer Krzonowski, Francois Pellegrino, Emmanuel Ferragne

\section{To cite this version:}

Jennifer Krzonowski, Francois Pellegrino, Emmanuel Ferragne. Étude acoustique de la production de voyelles de l'anglais par des apprenants francophones. Journées d'Études sur la parole, Jun 2018, Aix-en-Provence, France. pp.523-531, 10.21437/jep.2018-60 . hal-01960591

\section{HAL Id: hal-01960591 \\ https://hal.univ-lyon2.fr/hal-01960591}

Submitted on 19 Dec 2018

HAL is a multi-disciplinary open access archive for the deposit and dissemination of scientific research documents, whether they are published or not. The documents may come from teaching and research institutions in France or abroad, or from public or private research centers.
L'archive ouverte pluridisciplinaire HAL, est destinée au dépôt et à la diffusion de documents scientifiques de niveau recherche, publiés ou non, émanant des établissements d'enseignement et de recherche français ou étrangers, des laboratoires publics ou privés. 


\title{
Étude acoustique de la production de voyelles de l'anglais par des apprenants francophones
}

\author{
Jennifer Krzonowski ${ }^{1}$ François Pellegrino ${ }^{1}$ Emmanuel Ferragne ${ }^{2}$
}

(1) Laboratoire Dynamique du Langage, 14 avenue Berthelot, 69005 Lyon, France

(2) CLILLAC-ARP EA 3967 / Université Paris Diderot

jennifer.krzonowski@enrs.fr, françois.pellegrino@enrs.fr, emmanuel. ferragne@univ-paris-diderot.fr

\section{RESUME}

Les apprenants francophones tardifs de l'anglais présentent un accent étranger dont la littérature souligne qu'il est particulièrement marqué, en ce qui concerne les voyelles, pour $/ \mathrm{I} / \mathrm{,} / \mathrm{\Lambda} /$ et /æ/ qui seraient prononcées « à la française ». Nous avons étudié les paramètres acoustiques des voyelles /I/, /i:/, /æ/, / $/ \mathrm{h}$ et /a:/ de l'anglais produites par 38 locuteurs natifs de l'anglais et par 48 apprenants de langue maternelle française, ainsi que ceux des voyelles /a/, /e/, /i/ et /œ/ du français produites par ces mêmes apprenants. Les résultats montrent tout d'abord une plus grande variabilité des productions en L2 qu'en L1. De plus, les productions en L2 du contraste /i: - I/ présentent une grande confusion entre les deux catégories, alors que l'effet inverse est observé dans la région du [a]. Nos données montrent également que les apprenants produisent les contrastes de durée mais de manière moins marquée que les locuteurs natifs. Enfin, l'étude de la dynamique des formants pour les voyelles /i:/ et /i/ suppose une variabilité dans la diphtongaison du /i:/ produit par les natifs.

\section{ABSTRACT}

\section{An acoustic study of English vowels produced by French learners.}

Like every late L2-learner, French native speakers are characterized by a foreign accent. The literature emphasizes that the vowels $/ \mathrm{I} /, / N$ and $/ æ /$ in particular are pronounced "à la française". This study presents the acoustic parameters of the English vowels /I/, /i:/, /æ/, / $/ \mathrm{l}$ and /a:/ produced by 38 native English speakers and 48 French learners of English compared to those of the French vowels /a/, /e/, /i/ et /œ/ produced by the same learners. The results first show more variability in the L2 compared to L1 productions. Furthermore, we observe a great confusion between categories in L2 for the /i: - I/ contrast and the opposite effect in the region of the [a]. We show that L2 learners did produce duration contrasts but not to the same extent as the natives. Finally, the formant dynamic analysis of /i:/ and /i/ may suggest that the English native speakers do not all produce a diphthongal /i:/.

MOTS-CLES : acquisition d'une langue seconde, phonologie, voyelle, anglais langue seconde KEYWORDS: second language acquisition, phonology, vowel, English as an L2 


\section{Introduction}

Les apprenants de L2 tardifs sont souvent reconnaissables à leur accent étranger (Munro, 2008). Ce phénomène est bien décrit pour différentes combinaisons L1-L2 et pour différents contrastes phonologiques. L'explication théorique dominante attribue ces difficultés de production à un biais dans la perception de la L2 lié à la phonologie de la L1 et de ses liens avec la L2. Les modèles théoriques considèrent que l'acquisition de la production de la L2 suit celle de la perception. Une fois que les catégories de la L2 sont établies en perception, elles sont utilisées pour guider la production (Best \& Tyler, 2007; Flege, 1995; Schwartz \& Sprouse, 1996).

Dans l'enseignement des langues étrangères en France, la phonétique n'est que rarement abordée explicitement. Il a d'ailleurs été observé que des professeurs stagiaires présentaient de nombreuses difficultés de prononciation de l'anglais (Voise, 2010). En ce qui concerne les voyelles, cette étude rapporte notamment que plusieurs voyelles sont produites «à la française ». En effet, le /I/ anglais

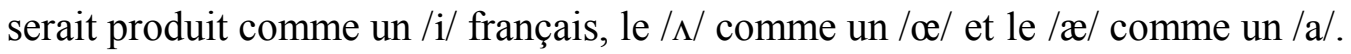

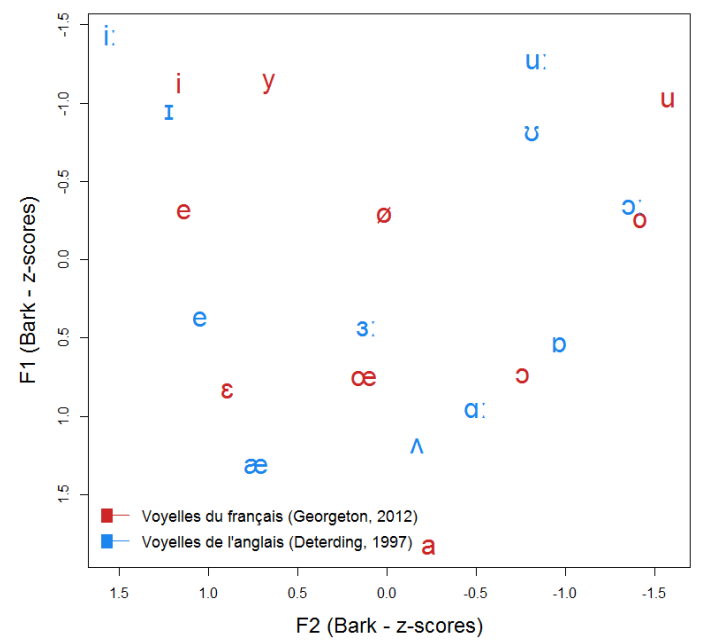

FigURE 1 : Voyelles du français (d'après Georgeton et al., 2012, en rouge) et monophtongues de l'anglais (d'après Deterding, 1997, en bleu), pour les femmes.

La comparaison des systèmes vocaliques (monophtongues) des deux langues (FIGURE 1), montre que dans la région du [a], une seule catégorie existe en français alors que l'anglais en présente trois, /æ/, / $/$ / et /a:/, qui se situent entre le /a/ français et le /œ/. Dans la région du [i], une seule catégorie est présente en français, alors que deux catégories occupent cette région en anglais, le /i:/ et le /I/. Outre le timbre des voyelles, la durée a une valeur phonologique en anglais, contrairement au français. De plus, ce contraste de durée produit en anglais une légère diphtongaison de certaines voyelles, et notamment du /i:/ (Collins \& Mees, 2013; Ferragne, 2008). Nous nous sommes donc intéressés, du point de vue acoustique, à la manière dont des apprenants francophones produisent ces voyelles et leurs voisines à la fois dans l'espace vocalique anglais et français. Deux approches ont été utilisées pour décrire des productions des voyelles de l'anglais en L1 et en L2, et du français en L1 : une approche statique pour l'ensemble des voyelles et une approche dynamique pour caractériser les voyelles /i:/ produites par des locuteurs natifs et des apprenants et la voyelle /i/ produite par les locuteurs francophones. 


\section{Méthodologie}

\subsection{Procédure expérimentale}

48 participants de langue maternelle française inscrits en 1 ère année de LEA ou LLCER anglais (dont 16 hommes) et 38 participants (dont 15 hommes) de langue maternelle anglaise originaires du Sud-Est de l'Angleterre ont participé à l'étude. Ils ont été enregistrés lors de tâches de lecture de mots isolées (Iverson, Pinet, \& Evans, 2012). Tous ont enregistré trois occurrences des voyelles

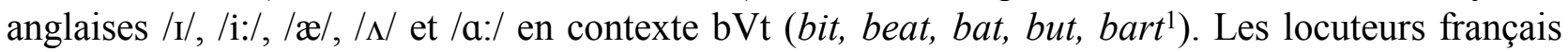
ont également enregistré trois occurrences des voyelles françaises /a/, /e/, /i/ et /œ/ en contexte $\mathrm{bV}(\mathrm{R})$ (bas, bée, bi, beurre) dans une tâche à part. Les enregistrements ont été réalisés avec le logiciel ROCme! au format PCM mono $44 \mathrm{kHz} 16$ bits.

\subsection{Analyse des données}

Des analyses acoustiques ont été réalisées avec le logiciel Praat (Boersma \& Weenink, 2017). Les voyelles ont été segmentées manuellement puis les trois premiers formants ont été mesurés semiautomatiquement sur toute la durée de la voyelle : l'estimation formantique de Praat superposée au spectrogramme était ajustée jusqu'à ce que l'estimation soit cohérente avec le spectrogramme. Cinq occurrences ont été retirées des analyses car les formants n'étaient pas visibles dans les spectrogrammes. Au total, le corpus comporte 1961 voyelles avec entre 113 et 142 occurrences par voyelles et par groupe. Les valeurs des deux premiers formants ont été extraites au milieu temporel des voyelles, transformées en Bark (Traunmüller, 1990) puis centrées-réduites par locuteur indépendamment pour chaque formant. Les durées des voyelles ont également été extraites.

\section{Résultats}

\subsection{Analyse statique des formants}

Les analyses du timbre des voyelles ont été réalisées séparément pour les hommes et les femmes. Dans les représentations graphiques qui suivent, les données des femmes sont représentées à gauche, celles des hommes à droite. Pour chacun des graphiques, les symboles phonétiques indiquent le barycentre de la catégorie, et les ellipses représentent l'intervalle de confiance au seuil de $95 \%$ des valeurs moyennes des formants pour les groupes de participants concernés. Des proportions de chevauchement entre les catégories voisines ont été mesurées en faisant le rapport entre l'aire de l'intersection par l'aire de l'union des deux catégories considérées.

1 L'anglais du Sud-Est de l'Angleterre est proche de la variété d'anglais enseignée en France, d'où cette restriction dans les critères d'inclusion. Il faut noter que dans cette variété, le $\mathrm{r}$ en coda ne se prononce pas. Ainsi bart se prononce [ba:t]. 


\subsubsection{Comparaison des voyelles anglaises et françaises produites par des natifs}
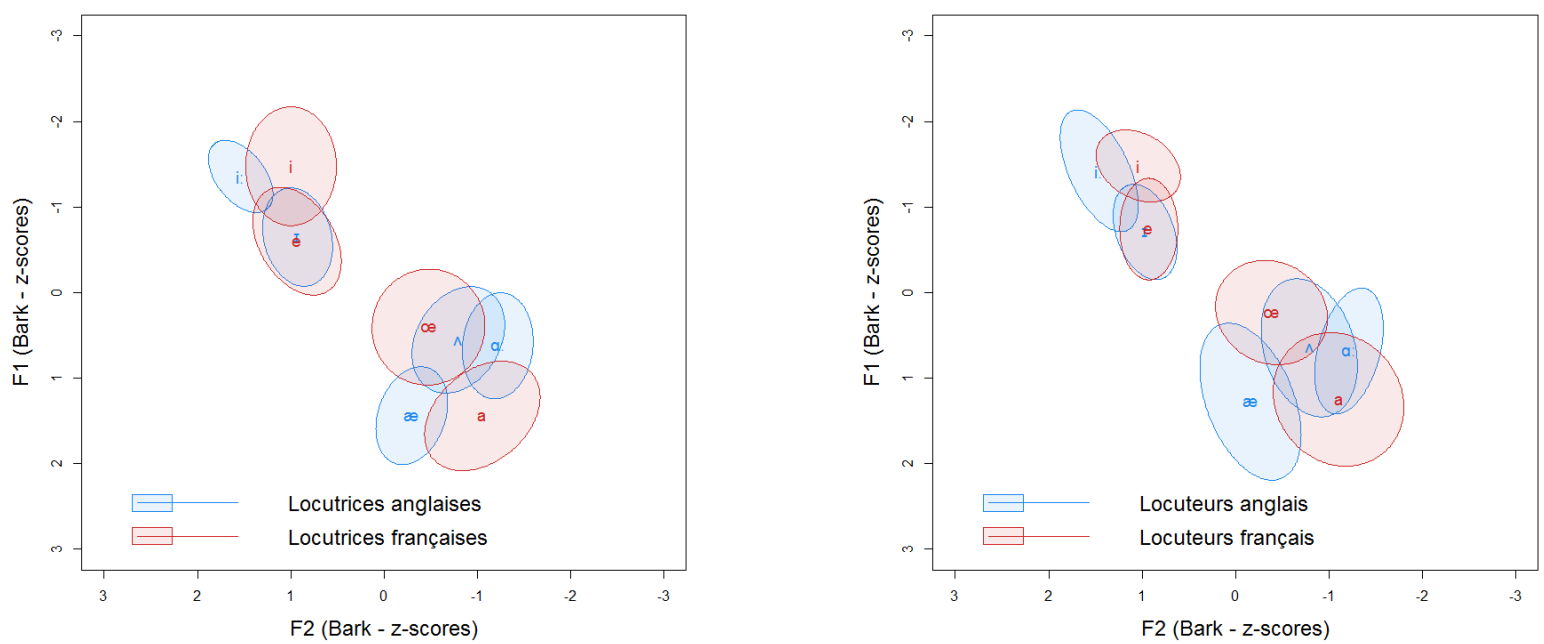

FIGURE 2 : Voyelles de l'anglais (en bleu) et du français (en rouge) produites par des locuteurs natifs, pour les femmes à gauche, pour les hommes à droite.

Une analyse de la variance sur les aires des ellipses pour chaque catégorie vocalique ne montre pas d'effet de la Langue, du Sexe des participants, ni d'interaction Langue $\times$ Sexe. Pour les femmes et les hommes, on constate que les aires des ellipses sont équivalentes en anglais et en français $\left(F_{(1,14)}=2.06, p>.05\right)$. Il semble donc que la variabilité intra-catégorielle produite par des locuteurs natifs soit équivalente d'une langue à l'autre.

Dans la région acoustique du [i], le /i/ français occupe un espace distinct des catégories anglaises : il est moins antérieur que le /i:/ anglais et plus fermé que /I/. Ainsi il partage peu d'espace acoustique avec ces catégories : $12 \%$ de chevauchement chez les femmes, $4 \%$ chez les hommes pour $/ \mathrm{I} / ; 9 \%$ chez les femmes et $15 \%$ chez les hommes pour /i:/. En revanche, le /e/ français occupe la même zone que le /I/ anglais avec $72 \%$ de superposition entre les deux catégories pour les femmes, $75 \%$ pour les hommes. On peut ainsi s'attendre à ce que les locuteurs français produisent cette voyelle avec les routines articulatoires du /e/ français.

Dans la région du [a], pour les hommes et les femmes, le /æ/ occupe une zone plus antérieure que les autres voyelles françaises et anglaises et ne partage ainsi que très peu son espace acoustique

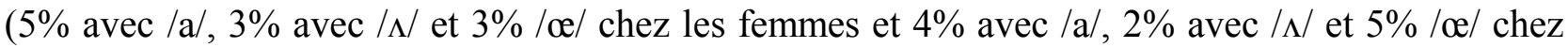
les hommes). Il en est de même pour le /a/ français chez les femmes, qui est plus ouvert que les voyelles $/ œ /, / \Lambda /$ et $/ \mathrm{a}: /$, plus postérieur que le /æ/ anglais et partage peu d'espace acoustique avec elles (3\% avec $/ \Lambda /, 11 \%$ avec $/ \mathrm{a}: /, 0 \%$ avec $/ œ /)$. Le profil est un peu différent chez les hommes puisque cette voyelle est moins ouverte et partage donc davantage d'espace avec les voyelles $/ \mathrm{A} /$ $(27 \%)$ et /a:/ (24\%). On peut enfin noter une grande confusion dans les espaces acoustiques des voyelles $/ œ /$ et $/ \Lambda /$ (41\% de chevauchement chez les femmes, $29 \%$ chez les hommes). On peut s'attendre ici à ce que les français réemploient les schémas articulatoires de cette voyelle pour produire le $/ \Lambda /$ de l'anglais comme observé dans l'étude de Voise (2010). On remarque enfin que parmi les voyelles de L1 étudiées dans les deux langues, les voyelles de l'anglais $/ \mathrm{h} / \mathrm{et} / \mathrm{a}$ :/ sont celles qui partagent les plus grandes proportions de leurs espaces acoustiques (23\% pour les femmes, $24 \%$ pour les hommes). 


\subsubsection{Comparaison des voyelles anglaises et françaises produites par des locuteurs français}
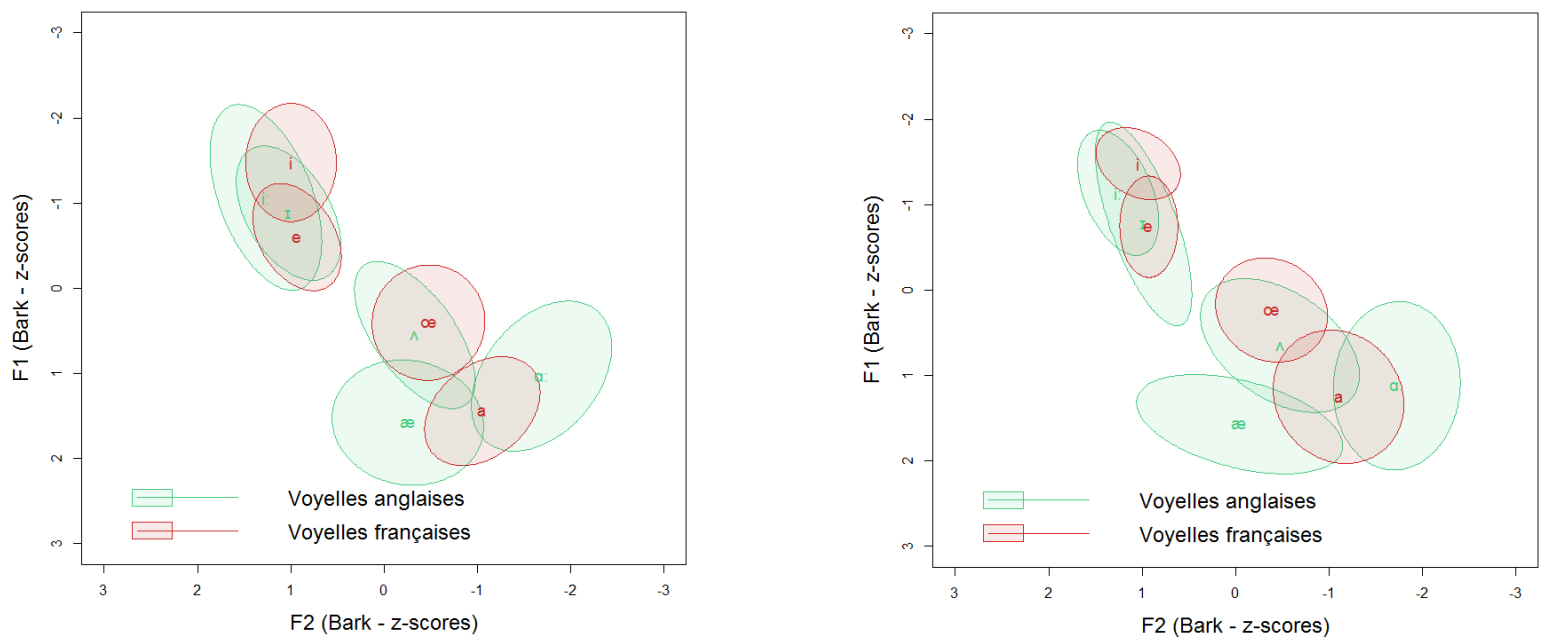

FIGURE 3 : Voyelles produites par les locuteurs français, en anglais (en vert) et en français (en rouge), pour les femmes à gauche, pour les hommes à droite.

Une analyse de la variance sur les aires des ellipses pour chaque catégorie vocalique montre cette fois un effet du facteur Langue $\left(F_{(1,14)}=9.40 ; p<.01\right)$, indiquant une plus grande variabilité des productions des locuteurs français lorsqu'ils produisent des sons de L2 que de L1 et conduisant à de forts chevauchements entre les différents espaces des voyelles.

Dans la région acoustique du [i], on remarque une forte confusion dans les espaces des voyelles anglaises /i:/ et /I/ (50\% pour les femmes, $41 \%$ pour les femmes) probablement due à la grande variabilité des productions. Néanmoins, on observe que la configuration des centres de catégories par rapport aux voyelles françaises semble tendre vers les cibles anglaises. En effet, le /i:/ est plus postérieur et plus ouvert que le /i/ français, le /I/ plus ouvert que le /i/ et se rapprochant ainsi du /e/.

Dans la région du [a], il est frappant de constater que les trois catégories anglaises sont produites dans des espaces acoustiques distincts avec peu de chevauchement entre les catégories $(<10 \%)$. Comme attendu, on observe une grande confusion entre les espaces $\mathrm{du} / \mathrm{c} /$ et $\mathrm{du} / \mathrm{A} /(52 \%$ pour les femmes, $40 \%$ pour les hommes). L'espace acoustique du /a/ chevauche les trois catégories de l'anglais mais les centres des catégories restent bien distincts.

\subsubsection{Comparaison des voyelles anglais produites en $\mathrm{L} 1$ et $\mathrm{L} 2$}

Une analyse de la variance réalisée sur les aires des ellipses indique ici encore que les productions des français en L2 présentent une plus forte variabilité que celles des anglais en L1 et ce pour toutes les voyelles $\left(F_{(1,16)}=69.31, p<.001\right)$. 

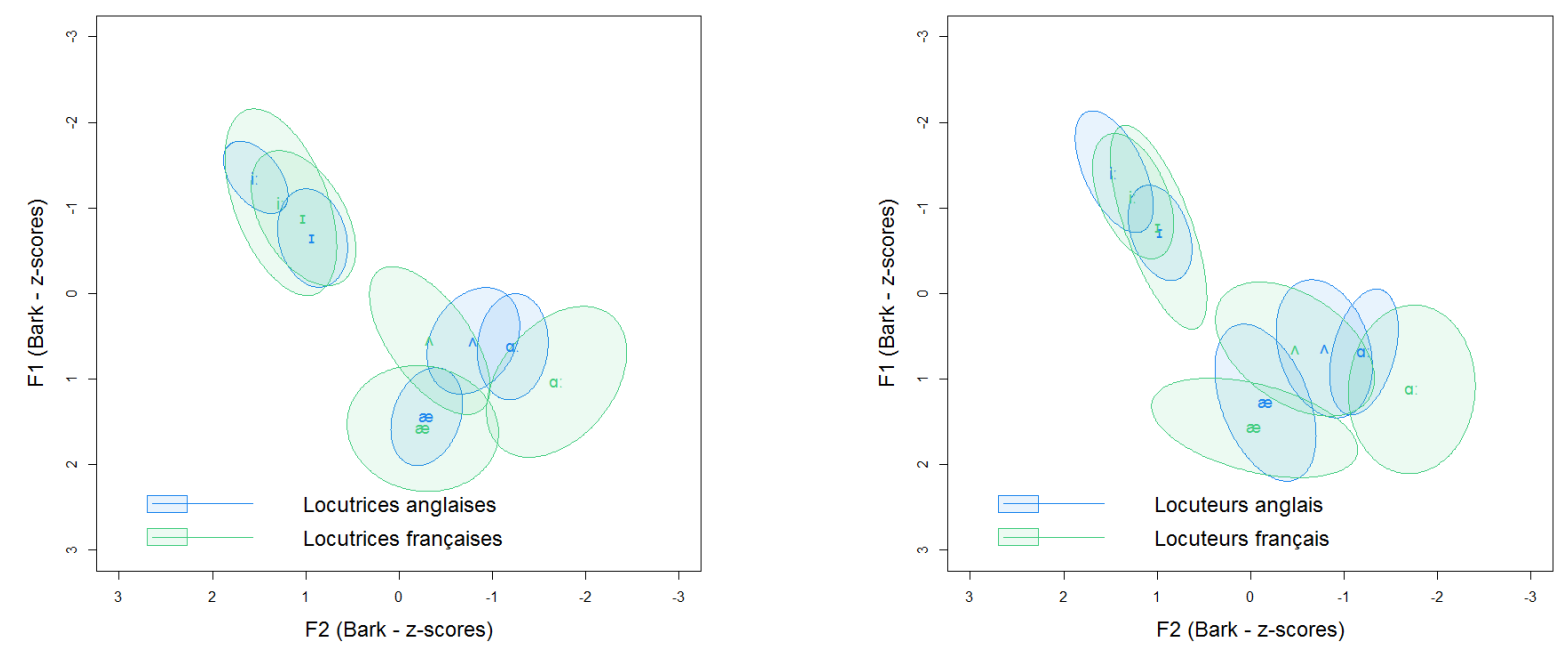

FIGURE 4: Voyelles anglaises produites par des locuteurs natifs (en bleu) ou de L2 (en vert), à gauche pour les femmes, à droite pour les hommes.

Dans la région du [i], on observe que les voyelles produites par les natifs sont plus distinctes que celles produites par les apprenants $(0 \%$ de chevauchement entre les deux catégories chez les femmes, $8 \%$ chez les hommes pour les natifs versus $50 \%$ pour les femmes, $41 \%$ pour les hommes chez les apprenants). De plus, on observe une plus grande distance acoustique entre les centres des catégories des natifs $(M=2.055)$ que ceux des apprenants $(M=1.94)$.

Dans la région du [a], on remarque le phénomène inverse : les voyelles des apprenants sont plus distinctes que celles des locuteurs natifs. On observe un chevauchement moins important entre les trois catégories produites par les locuteurs francophones que par les locuteurs natifs, de plus, les distances acoustiques dans F1/F2 entre les centres des catégories des francophones sont plus grandes que celles des locuteurs natifs. Il semble que dans cette région, les participants français utilisent davantage le timbre des voyelles pour produire des voyelles qui soient à la fois distinctes entre elles mais aussi pour le /æ/ et le /a:/ différentes du /a/ français. Au sujet du $/ \Lambda /$, on note que les productions des apprenants s'étendent sur une région bien plus centrale que les productions des natifs, ce qui vient appuyer l'idée que les apprenants utilisent le /œ/ comme cible articulatoire.

\subsection{Analyse des durées des voyelles}

Les voyelles de l'anglais présentent des différences de durées significatives $\left(F_{(4,148)}=258.57, p<.0001\right)$. Les voyelles $/ æ /(290 \mathrm{~ms})$, /a:/ $(340 \mathrm{~ms})$ et $/ \mathrm{i}: /(287 \mathrm{~ms})$ sont plus longues que les voyelles $/ \Lambda /(172 \mathrm{~ms})$ et $/ \mathrm{I} /(159 \mathrm{~ms})$ (FIGURE 5 , en bleu). Les voyelles du français présentent également des différences de durées significatives $\left(F_{(3,141)}=55.80, p<.001\right)$, mais cette différence n'est portée que par la voyelle /œ/ produite en contexte bVR, qui a pour effet d'allonger la durée de la voyelle (Léon, 2005) (FIgURE 5, en rouge). Ainsi les voyelles du français ont des durées similaires aux voyelles courtes de l'anglais (sauf le /œ/ qui est plus long) et toutes sont significativement plus courtes que les voyelles longues de l'anglais (y compris /œ/). 


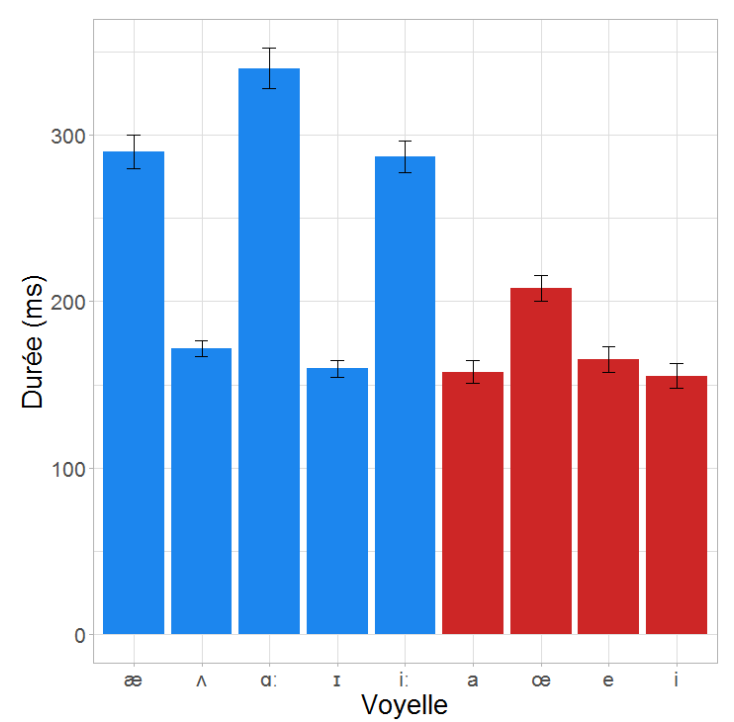

FIGURE 5 : Durées moyennes des voyelles anglaises (en bleu) et françaises (en rouge) produites par des locuteurs de L1.

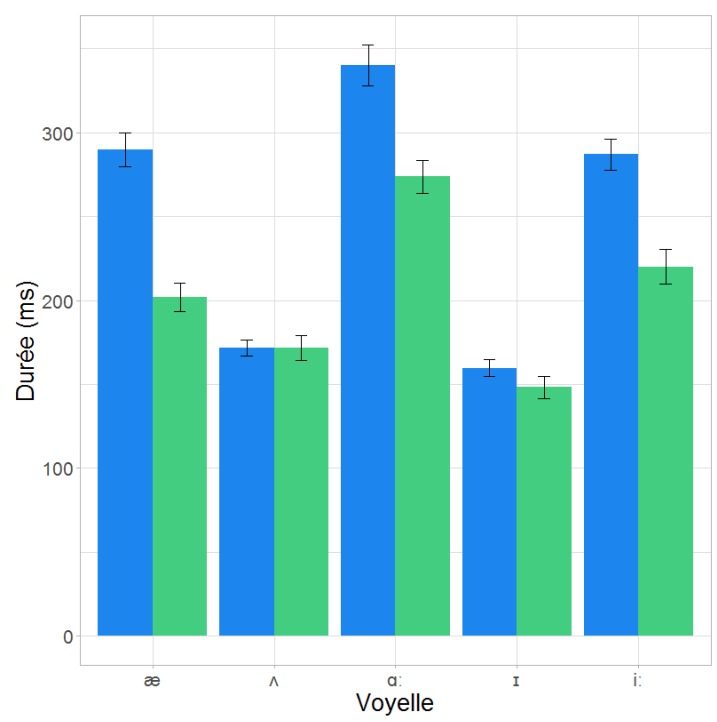

FIGURE 6 : Durées moyennes des voyelles anglaises produites par des locuteurs de L1 (en bleu) et de L2 (en vert).

Lorsque les apprenants français produisent les voyelles anglaises (FIGURE 6, en vert), les durées des voyelles courtes $(/ \mathrm{K} /$ et $/ \mathrm{I} /)$ sont similaires aux durées de ces mêmes voyelles produites par des locuteurs natifs (en bleu) $\left(F_{(1,84)}=1.66, p>.05\right)$. En revanche, pour les voyelles longues, on observe que les durées des voyelles produites par les locuteurs français sont significativement plus courtes que celles produites par les locuteurs natifs $\left(F_{(1,84)}=7.732, p<.001\right)$. Cependant, pour ces mêmes voyelles, on observe bien un allongement de la durée par rapport aux voyelles françaises $\left(F_{(1,335)}=14.56, p<.001\right)$. Ainsi, il semblerait qu'en L2, les apprenants français soient capables de produire un contraste de durée bien qu'il n'en existe pas dans leur L1.

\subsection{Analyse dynamique des voyelles /i:/ et /i/}

Suivant Williams \& Escudero (2014), les 80\% centraux de chaque courbe de F2 des voyelles /i :/ et /i/ produites par des natifs et des voyelles /i:/ produites par les apprenants ont été rééchantillonnées afin d'avoir 30 points. Les coefficients 2 et 3 (DCT2 et DCT3) d'une transformée en cosinus discrète ont été calculés. Ces coefficients permettent de caractériser les trajectoires de formants. Les comparaisons de différents modèles d'analyse discriminante linéaire montrent que la valeur des deux premiers formants seuls prise au milieu temporel suffit à distinguer les voyelles /i:/ $(97 \%)$ et /i/ (90\%) produites par des natifs lorsque ces seules voyelles sont intégrées dans l'analyse. Un modèle incluant uniquement DCT2 classifie correctement les /i/ français (87\%) mais pas les /i:/ anglais (46\%), ce qui indique que ce paramètre est plus pertinent pour classifier les /i/ français que les /i:/ anglais, probablement à cause de la non-systématicité de la diphtongaison du /i:/ anglais. Le meilleur modèle pour classifier ces voyelles natives a été ensuite utilisé pour classifier les /i:/ des apprenants. Ce modèle porte sur F1, F2, la durée et DCT2. Ce modèle classe $58 \%$ des voyelles de L2 comme des /i:/ natif et $42 \%$ comme des/i/ français. Bien que non significatif, $\left(X^{2}(1)=3.36, p=.067\right)$, ce résultat semble indiquer qu'au moins une partie des /i:/ produits par les apprenants présentent des paramètres acoustiques semblables à ceux des voyelles produites par les natifs, y compris des paramètres dynamiques. 


\section{Discussion}

Pour les voyelles étudiées, la comparaison des espaces vocaliques - anglais natif, français natif et anglais L2 - montre tout d'abord une plus grande variabilité des voyelles produites en L2. Ceci peut s'expliquer par le fait qu'en L2 les cibles articulatoires ne sont pas clairement établies chez les apprenants (Gick, Bernhardt, Bacsfalvi, \& Wilson, 2008).

Pour les contrastes de l'anglais /i: - I/ et / $\mathrm{-a}$ :/, qui portent à la fois sur le timbre et la durée, nous avons observé des phénomènes différents dans les productions des locuteurs natifs. En effet, les catégories /i:/ et /I/ sont produites par les natifs avec une plus grande distance acoustique entre les centres de catégories que ne le sont les voyelles $/ \Lambda /$ et $/ \mathrm{a}: /$. Le plus grand chevauchement des aires des catégories $/ \Lambda /$ et $/ \mathrm{a}$ :/ résultant est contrebalancé par un contraste de durée plus marqué pour ce contraste. Chez les apprenants, ce schéma est différent : ils produisent le contraste /i: - I/ avec une faible distance acoustique entre les catégories et donc un fort chevauchement entre leurs aires, alors qu'ils produisent le contraste $/ \Lambda-\mathrm{a}$ / avec une grande distance entre les catégories et un faible chevauchement entre leurs aires, et ce, malgré une grande variabilité intra-catégorielle. Il semblerait que, pour les voyelles de la région du [a] (/æ/ compris), les apprenants cherchent à produire des voyelles qui se distinguent du point de vue du timbre, à la fois entre elles, mais aussi $\mathrm{du} / \mathrm{a} /$ français. Cette observation renvoie au phénomène de dissimilation décrit dans le Speech Language Model de Flege (1995). Selon ce modèle, la probabilité de formation d'une catégorie phonétique augmente avec la dissimilarité entre un son de L2 et le plus proche voisin en L1. En l'absence de formation de nouvelle catégorie, les propriétés d'un son L2 et d'un son L1 voisin peuvent fusionner pour former une catégorie «composite ». Les éléments phonétiques des deux inventaires coexistent dans un espace phonologique commun et interagissent les uns avec les autres conduisant parfois à des déviations (assimilation ou dissimilation) des catégories voisines de L1, L2 et L1/L2 pour maintenir les contrastes phonétiques.

Pour le contraste /i: - I/, les apprenants semblent au contraire présenter des difficultés à produire des voyelles distinctes du point de vue du timbre, ne produisant quasiment qu'un contraste de durée. Bien que des précautions aient été prises lors de la présentation des consignes (le mot était accompagné du symbole phonétique de la voyelle), on peut imaginer pour ce contraste que les participants francophones aient été influencés par le système orthographique de leur L1 selon lequel la graphie du mot «bit» induit la production d'un [i] (Nimz, 2016). Selon cette interprétation, la réalisation $\mathrm{du} / \mathrm{I} /$ tendrait à se rapprocher de celle $\mathrm{du} / \mathrm{i} /$ pour des raisons orthographiques, et ce malgré le fait que le /e/ soit acoustiquement un meilleur support articulatoire pour la réalisation $\mathrm{du} / \mathrm{I} /$. Au sujet de la voyelle $/ \mathrm{N} /$ plus particulièrement, on a pu remarquer que la distribution des voyelles produites par les apprenants s'étendait de manière plus importante que chez les natifs en direction de la région du /œ/, ce qui confirme que cette voyelle pourrait en effet servir de support articulatoire aux apprenants francophones.

En ce qui concerne la durée des voyelles, nos données montrent que, bien que la durée ne soit pas contrastive en français, les apprenants francophones produisent des voyelles longues de l'anglais plus longues que les voyelles françaises même si celles-ci restent moins longues que celles des locuteurs natifs.

L'analyse dynamique pour les voyelles /i:/ de l'anglais et /i/ du français montre tout d'abord que les indices statiques des deux premiers formants seuls suffisent à catégoriser ces voyelles alors 
qu'un indice dynamique seul n'est pertinent que pour les voyelles françaises, ce qui pourrait indiquer une certaine variabilité dans les productions des /i:/ par les natifs (Ferragne, 2008). Enfin, une partie des productions des apprenants présentent des paramètres similaires aux voyelles natives.

\section{Remerciements}

Ce travail est soutenu par la subvention de recherche de l'IUF d'E. Ferragne et le LabEx ASLAN de 1'Université de Lyon (ANR-10-LABX-0081).

\section{Références}

Best, C. T., \& TYLER, M. D. (2007). Nonnative and second-language speech perception: Commonalities and complementarities. Language experience in second language speech learning: In honor of James Emil Flege, 1334.

Boersma, P., \& WeEninK, D. Praat: doing phonetics by computer. (Version 6.0.26).

Collins, B., \& Mees, I. M. (2013). Practical Phonetics and Phonology: A Resource Book for Students. Routledge.

Deterding, D. (1997). The Formants of Monophthong Vowels in Standard Southern British English Pronunciation. Journal of the International Phonetic Association, 27(1-2), 47-55.

FerRagne, E. (2008). Etude Phonétique des Dialectes Modernes de l'Anglais des Iles Britanniques: Vers l'Identification Automatique du Dialecte. Unpublished doctoral thesis, Université Lyon, 2.

Flege, J. E. (1995). Second language speech learning: Theory, findings, and problems. Speech perception and linguistic experience: Issues in cross-language research, 92, 233-277.

Georgeton, L., Paillereau, N., Landron, S., GaO, J., \& Kamiyama, T. (2012). Analyse formantique des voyelles orales du français en contexte isolé : à la recherche d'une référence pour les apprenants de FLE (p. 145 -152). Présenté à Conférence conjointe JEP-TALN-RECITAL 2012.

GiCK, B., BERNHARDT, B., BACSFALVI, P., \& WilsON, I. (2008). 11. Ultrasound imaging applications in second language acquisition. In J. G. Hansen Edwards \& M. L. Zampini (Éd.), Studies in Bilingualism (Vol. 36, p. 309-322). Amsterdam: John Benjamins Publishing Company.

IVERSON, P., Pinet, M., \& Evans, B. G. (2012). Auditory training for experienced and inexperienced second-language learners: Native French speakers learning English vowels. Applied Psycholinguistics, 33(1), 145-160.

LÉON, P. R. (2005). Phonétisme et prononciations du français. A. Colin.

MunRo, M. J. (2008). 7. Foreign accent and speech intelligibility. In J. G. Hansen Edwards \& M. L. Zampini (Éd.), Studies in Bilingualism (Vol. 36, p. 193-218). Amsterdam: John Benjamins Publishing Company.

NiMZ, K. (2016). Sound perception and production in a foreign language.

SchwartZ, B. D., \& Sprouse, R. A. (1996). L2 cognitive states and the Full Transfer/Full Access model. Second language research, 12(1), 40-72.

TRAUnMÜLleR, H. (1990). Analytical expressions for the tonotopic sensory scale. The Journal of the Acoustical Society of America, 88(1), 97-100.

VoISE, A.-M. (2010). Enseigner la phonologie de l'anglais aux futurs professeurs du primaire. Recherche et pratiques pédagogiques en langues de spécialité. Cahiers de l'Apliut, (Vol. XXIX N 2), 11-24.

Williams, D., \& Escudero, P. (2014). A cross-dialectal acoustic comparison of vowels in Northern and Southern British English. The Journal of the acoustical society of America, 136(5), 2751-2761. 